Milk Drop (Tipat Halav) Nurses Preparedness for Coping with Continuous War and Terrorism Marc Gelkopf; ${ }^{1}$ Rony Berger ${ }^{2}$

1. Department of Community Mental Health, Faculty of Social Welfare and Health Sciences, University of Haifa, Haifa, Israel

2. NATAL-The Israel Trauma Center for Victims of Terror and War, Ben Gurion University, Israel

Introduction: Research has suggested infants were at risk for the development of post-traumatic symptomatology in situations of major disaster and in the context of the ongoing, violent Israeli-Palestinian conflict. Research also has shown that caretaker stress was directly related to infant stress. Milk drop nurses (MDN) are among the first professionals that observe, diagnose, and provide psychosocial information and direct infants and their mothers for further medical treatment after birth. In times of war and terrorism, it is imperative that MDNs have adequate knowledge to help parents cope with the situation, strengthen their resilience, impart soothing and modulation parental skills for their infants, and direct mothers and infants to mental health treatment when needed. Furthermore, it is important to teach MDN self-soothing and stress management strategies that will enhance their ability to better cope with stressful and even traumatic situations.

Methods: This study consisted of a randomly controlled study assessing a 12-session, manualized intervention aimed at training MDNs to identify infants and parents at risk for traumatic reactions, help parents cope with disaster-related situations, develop work-related self-efficacy, and reduce the risk of secondary traumatization. Eighty MDNs (42 study and 38 waiting list) were assessed, before and three months after the intervention on measures of secondary traumatization, work burnout, work-related selfefficacy, hope, sense of control, and self appraisal.

Results: Using repeated measure ANOVA, a significant and positive impact of group $x$ time on all assessed measures was found.

Conclusions: Imparting adequate training to MDNs is an effective way to improve work efficacy, strengthen resilience, and protect against secondary traumatization. Although not assessed in this study, such training might strengthen the resilience of parents and their newly born infants in cases of war and terror.

Keywords: infants; Milk Drop Nurses; parents; terrorism; training; trauma

Prebosp Disaster Med

\section{Palliative Care Considerations in Mass-Casualty Events with Scarce Resources}

Anne M. Wilkinson, PbD; Marianne Matzo, PbD, $R N$; Joanne Lynn, MD; Maria Gatto, RN; Sally Phillips

Edith Cowan University, School of Nursing, Midwifery and Postgraduate Medicine, Perth, Australia

Catastrophic mass-casualty events (MCEs), such as pandemic influenza outbreaks or large-scale, terrorism-related events, could yield thousands of victims whose needs would overwhelm local and regional healthcare systems, personnel, and resources. Such conditions would require the deployment of scarce resources in a manner different from the more common single-event disaster. The purpose of this presentation is to offer an introduction to the topic of palliative care during a MCE and to review the major findings for a federally funded planning guide that examined palliative care issues associated with providing medical care during MCEs, when resources are scarce. The focus of this presentation is on the role of palliative care in the support of individuals not expected to survive and recommendations of specific actions for a coordinated disaster response plan. Semi-structured telephone discussions with disaster management experts and an expert group meeting identified issues, roles, responsibilities, procedures, and resources offering benefits of integrating palliative care into disaster planning and response. The investigations identified five domains of concern, along with guidance: (1) the role of palliative care in a MCE with resulting scarce resources; (2) the triage and ensuing treatment decisions for those "likely to die"; (3) the critical palliative care services to provide, along with the personnel and settings; (4) the pragmatic plans needed for ensuring training, supplies, and organizational or jurisdictional arrangements; and (5) unusual issues affecting palliative care under MCE scenarios. Palliative care minimizes the suffering of those who die, ensures comfort, addresses the needs of those who will not survive, and may also free up resources to optimize survival of others. Planning to provide palliative care during MCEs should be part of the current state and local disaster planning/training guidelines, protocols, and activities.

Keywords: mass-casualty incident; pallitative care; scarce resources Prebosp Dissaster Med

\section{Emergency Preparedness of Local Authorities: General Model and the Israeli Case Alex Altshuler}

Ben-Gurion University of the Negev, Israel

Introduction: While wars have not been rare in Israeli history and its immediate past, the massive involvement of the civil population and its local administrations in war-caused disaster is a relatively new experience. To meet the need for theoretical models for disaster research and valid empirical tools for examination of emergency preparedness for warcaused disaster, the current study examined emergency preparedness of local authorities throughout Israel for this kind of disaster and the factors that may help to predict the level of preparedness.

Methods: A structured questionnaire developed specifically for this study included 74 items, divided into nine subquestionnaires that were used for measurement of all of the research variables. The dependent variable-preparedness level-was constructed on the basis of the official obligatory requirements of the Home Front Command and the Union of Local Authorities in Israel. The questionnaires were distributed in May-July 2008 among 177 local authorities' chief emergency managers throughout Israel (87.6\% of the total population of these position holders in Israel, but not of the total number of local authorities, since not all of them have a professional emergency manager), 
using a random sampling technique. The response rate was $80.8 \%$ (143 out of 177 ).

Results: The following factors predicted the preparedness level: (1) joint activity of local emergency managers with governmental and non-governmental stakeholders; (2) socio-economic situation in a local community; (3) collective efficacy; and (4) the city's previous war exposure. However, risk perception, population size, ethnic composition of a local community, and financial resources were not significant in the framework of the comprehensive model.

Conclusions: Identification of factors that contribute to the preparedness level has important practical implications. The validation of the measurement instruments is important theoretically. Enhancement of significant contributing factors may enable an increase in the level of local preparedness.

Keywords: emergencies; Israel; local authorities; preparedness; responders

Prehosp Disaster Med

\section{Community Stress Treatment Centers: A Novel} Concept of Civilian "Front Line"Treatment for Anxiety and Acute Stress Reaction of Civilians under Continuous Rocket Attacks during the Second Lebanon War

Zohar Rubinstein; ${ }^{1,2}$ Yackov Polakevitz;, 3

Bella Ben Gershon,; Gadi Lubin; ${ }^{4}$ Yaron Bar-Dayan ${ }^{5,6}$

1. Mental Health Section, Medical Department, Home Front Command, Israel Defense Forces, Israel

2. The Multidisciplinary Graduate Program for Emergency \& Disaster Management, The School of Public Health, Sackler Faculty of Medicine, Tel Aviv University, Ramat Aviv, Israel

3. Mental Health Services, Ministry of Health, Israel

4. Mental Health Department, the Medical Force, Israel Defense Forces, Israel

5. Bar Ilan University, Ramat Gan, Israel

6. Ben Gurion University of the Negev, Beersheba, Israel

This study describes the treatment of anxiety and acute stress reaction (ASR) civilian casualties under rocket bombardments during Lebanon War II during the summer of 2006, in community stress treatment centers (CSTCs) (then called resilience centers) erected ad hoc, by a joint effort of the Mental Dealth Branch of the Israel Defense Forces (IDF) Home Front Command Medical Department, and the Mental Health Services of the Ministry of Health $(\mathrm{MOH})$ and the Emergency Wing of the MOH.

A total of 536 casualties were admitted to these centers. Only 18 were evacuated to the local medical centers. The majority were released to their homes after some 40 to 200 minutes of immediate treatment according to a protocol.

The symptoms of the casualties included anxiety (90\%), fear $(7 \%)$, and sleep disturbance (1\%). Ninety-two percent were diagnosed as suffering from ASR. The treatment included counseling (80\%), ventilation (9\%), relaxation (3\%), non-verbal intervention (3\%), supply of basic needs $(1 \%)$, medication (1\%), and evacuation to hospitals (3\%).

Conclusions: The ASR and anxiety are the most common injuries seen during a missile bombardment of civil population. The CSTCs are able to provide immediate treatment to most of those casualties and in most of the cases to prevent the need for evacuation to the hospitals. Thus, they enable saving of evacuation resources during air bombardment and reduce the load on hospital emergency rooms.

Disaster preparedness should include the establishments of such centers as one of the components of the mental health system response to terrorist and air-attack scenarios. Keywords: acute stress reaction; anxiety; community stress treatment centers; mental health; rocket bombardments Prebosp Disaster Med

Treatment of Walking Patients during Chemical Warfare: Presenting the Examination and Treatment Center

Farfel Alon, MD; Bar Ariel, MD

Israel

A review of a missile strike with chemical weapons (an organic phosphorus) predicts many casualties, most of whom will be ambulatory. A large number of victims suffering from anxiety also is expected. These injuries usually do not require hospitalization for medical treatment, and can be provided with care outside of hospital supervision. However, these patients add to those who are treated unnecessarily with atropine and to those with exacerbation of an existing disease for various reasons. The arrival of ambulatory casualties to hospitals could affect the ability of hospitals to treat casualties with medium to severe injuries.

A unique solution was developed to manage these casualties, including: (1) decontamination (to remove possible remnants of chemical warfare material); (2) systemic treatment with antidotes until reaching a state of atropinization; and (3) supervision for several hours. Treatment of anxiety victims involves reassurance and assistance in order for them to cope with the tragic events they experienced. Therapy is based on conversations and physical activity provided by psychologists and social workers, rather than medication. Any patient who deteriorates despite optimal treatment requires a referral for further treatment in a hospital.

To address this challenge the Examination and Treatment Center (MABAT) was established. The MABAT has several aspects that are unique, the first being the combination of military and civilian medical personnel. The military has an advantage in speed, logistics, and resilience; therefore, the management of the MABAT is the responsibility of the Home Front Command. The medical civilian Health Maintenance Organizations provide most of the professional work force. The MABATs are established in country clubs because these facilities can provide the many necessary showers needed for decontamination.

Last year, the Home Front Command in cooperation with the Ministry of Health performed several drills combining MABATs with hospital training. The MABAT is a unique solution because it is an example of cooperation between military and civilian medical systems that creates an unusual synergy to an unusual situation.

Keywords: ambulatory; chemical warfare; civilian medical; cooperation; decontamination; military

Prebosp Disaster Med 